\title{
Kinetics of cytochrome P450 enzymes for metabolism of sodium tanshinone IIA sulfonate in vitro
}

\author{
Dong-sheng Ouyang 1,2, Wei-hua Huang ${ }^{1}$, Dan Chen ', Wei Zhang ${ }^{1}$, Zhi-rong Tan', Jing-bo Peng',
} Yi-cheng Wang ${ }^{2}$, Ying Guo ${ }^{2}$, Dong-li Hu², Jian Xiao ${ }^{3}$ and Yao Chen ${ }^{1,2^{*}}$

\begin{abstract}
Background: Sodium tanshinone IIA sulfonate (STS) is a water-soluble derivative of tanshinone IIA for treating cardiovascular disorders. The roles of cytochrome P450 enzymes (CYPS) in the metabolism of STS have remained unclear. This study aims to screen the main CYPs for metabolism of STS and study their interactions in vitro.

Methods: Seven major CYPS were screened for metabolism of STS by human liver microsomes (HLMs) or recombinant CYP isoforms. Phenacetin (CYP1A2), coumarin (CYP2A6), tolbutamide (CYP2C9), metoprolol (CYP2D6), chlorzoxazone (CYP2E1), S-mephenytoin (CYP2C19), and midazolam (CYP3A4) were used as probe substrates to determine the potential of STS in affecting CYP-mediated phase I metabolism in humans. Enzyme kinetic studies were performed to investigate the modes of inhibition of the enzyme-substrate interactions by GraphPad Prism Enzyme Kinetic 5 Demo software.

Results: Sodium tanshinone IIA sulfonate inhibited the activity of CYP3A4 in a dose-dependent manner by the HLMs and CYP3A4 isoform. The $K_{m}$ and $V_{\text {max }}$ values of STS were $54.8 \pm 14.6 \mu \mathrm{M}$ and $0.9 \pm 0.1 \mathrm{nmol} / \mathrm{mg} \mathrm{protein} / \mathrm{min}$, respectively, for the HLMs and $7.5 \pm 1.4 \mu \mathrm{M}$ and $6.8 \pm 0.3 \mathrm{nmol} / \mathrm{nmol} \mathrm{P} 450 / \mathrm{min}$, respectively, for CYP3A4. CYP1A2, CYP2A6, CYP2C9, CYP2D6, CYP2E1, and CYP2C19 showed minimal or no effects on the metabolism of STS.

Conclusion: This in vitro study showed that STS mainly inhibited the activities of CYP3A4.
\end{abstract}

\section{Background}

Tanshinone IIA (Fig. 1a) is one of the main extracts from Salvia miltiorrhiza (Danshen) for treating cardiovascular disorders [1]. Tanshinone IIA is the most effective fat-soluble monomer extracted from Danshen, with anti-inflammatory [2], antitumor [3], antioxidative [4, 5], and antiplatelet aggregation activities [6, 7]. However, as clinical use of tanshinone IIA is limited by its poor water solubility, sodium tanshinone IIA sulfonate (STS) (Fig. 1b) has been developed through sulfonation with greater water-soluble characteristics and efficacies than tanshinone IIA [8], and has been used in injection with typical doses of $40-80 \mathrm{mg} / \mathrm{d}$ for cardioprotective [9,

\footnotetext{
*Correspondence: cbohua@163.com

${ }^{1}$ Department of Clinical Pharmacology, Xiangya Hospital, Central South University, 110 Xiangya Road, Changsha 410078, Hunan, China

Full list of author information is available at the end of the article
}

10], anti-cardiomyocyte hypertrophy [11], and antiviral effects [12-14]. To date, only several studies reported the pharmacokinetics parameters of STS in rats after injection. The metabolism rate of STS in rats was fast, with $t_{1 / 2}$ of $<0.9 \mathrm{~h}$, and at $4 \mathrm{~h}$ after the injection, the plasma concentration of STS in rats did not exceed $20 \mathrm{ng} / \mathrm{mL}[15$, 16].

CYPs comprise the most important phase I drugmetabolizing enzyme system, and are responsible for the metabolism of a variety of xenobiotics [17]. Yang et al. [18] reported that tanshinone (containing $20 \%$ tanshinone IIA) could significantly increase (2-9.5-fold) the activities of CYP1A1, CYP1A2, and CYP2B1, while inhibiting (1.9-fold) the activity of CYP2E1 in rats. Ueng et al. [19] reported that tanshinone IIA could decrease 7-ethoxyresorufin O-deethylation (EROD) and 7-methoxyresorufin O-demethylation (MROD) activities in 
<smiles>Cc1coc2c1C(=O)C(=O)c1c-2ccc2c1CCCC2(C)C</smiles>

a Tanshinone II A, $\mathrm{C}_{19} \mathrm{H}_{18} \mathrm{O}_{3}$<smiles></smiles>

b Sodium tanshinone II A sulfonate (STS), $\mathrm{C}_{19} \mathrm{H}_{17} \mathrm{NaO}_{6} \mathrm{~S}$

Fig. 1 Chemical structures of tanshinone IIA (a) and STS (b)

human liver microsomes (HLMs), and indicated that tanshinone IIA possessed the highest selectivity for inhibition of CYP1A2.The same authors further demonstrated that tanshinone IIA induced hepatic Cyp1A2 by increasing the expression levels of Cyp1A2 mRNA and protein in mice [20]. Liu et al. [21] reported that CYP2A6 was the specific isozyme responsible for hydroxyl metabolism of tanshinone IIA in HLMs. Although many experiments related to tanshinone IIA have been performed, the question of which CYP is responsible for the metabolism of STS has remained unclear.

The difference in molecular structure between STS and tanshinone IIA is only a sulfonic acid group at the C-16 position [22]. Although STS has a similar molecular structure to tanshinone IIA, the metabolism of STS and interactions with CYPs might differ from those of tanshinone IIA [16]. In our previous study, STS significantly increased the activity of CYP1A2 by $41.1 \%$ in healthy volunteers [23], and the possible mechanism might be the induction of CYP1A2 expression by tanshinone IIA transformed from STS in vivo. Which CYP participated in the transformation of STS, and related information is still critical.

As CYP1A2 (13\%), CYP2A6 (4\%), CYP2D6 (2\%), CYP2E1 (7 \%), CYP3A4 (30 \%), CYP2C19 (8 \%) and CYP2C9 $(20 \%)$ comprise $70-80 \%$ of the hepatic CYPs in humans and are responsible for more than $95 \%$ of clinical drug metabolism [17]. This study aims to screen the main CYPs for metabolism of STS and study their interactions in vitro.

\section{Methods}

Enzymes and chemicals

Recombinant human CYP enzymes (bactosomes), pooled human liver microsomes (HLMs) from ten individual donors (bactosomes), and $\beta$-nicotinamide adenine dinucleotide 2'-phosphate reduced tetrasodium salt hydrate (NADPH) were purchased from Cypex Ltd. (UK) and stored at $-80{ }^{\circ} \mathrm{C}$. STS $\left(\mathrm{C}_{19} \mathrm{H}_{17} \mathrm{NaO}_{6} \mathrm{~S}\right.$; MW: 396.39; assay: $\geq 98 \%$ ) was obtained from Sigma-Aldrich (China). Furafylline (FUR), trans-2-phenylcyclopropylamine hydrochloride (TRA), ketoconazole (KET), sulfaphenazole (SUL), quinidine (QUI), chlormethiazole hydrochloride (CHL), and ticlopidine hydrochloride (TIC) were purchased from the National Institutes for Food and Drug Control (China). Phenacetin, coumarin, midazolam, tolbutamide, S-mephenytoin, metoprolol, chlorzoxazone, and standards for their metabolites, including acetaminophen, 7-hydroxyl coumarin, 1-hydroxyl midazolam, 4-hydroxyl tolbutamide, 4-hydroxyl mephenytoin, $\alpha$-hydroxyl metoprolol, 6-hydroxyl chlorzoxazone, and irbesartan (internal standard) were purchased from Sigma-Aldrich (China). All other chemicals and solvents were of high-performance liquid chromatography (HPLC) grade.

\section{Apparatus and operation conditions}

The concentrations of the CYP substrates and their metabolites were quantified using a Waters 2695 Separation Module HPLC System (Waters Corp., USA) coupled to a Quattro Micro ${ }^{\mathrm{TM}}$ API Triple Quadrupole Tandem Mass Spectrometer (Waters Corp., USA) with an electrospray ionization source. The samples were separated on a HYPURITY $\mathrm{C}_{18}$ column $(150 \times 2.1 \mathrm{~mm}$; internal diameter: $5 \mu \mathrm{m}$; Thermo, USA) with a $\mathrm{C}_{18}$ security guard column $(4.0 \times 3.0 \mathrm{~mm}$; internal diameter: $5 \mu \mathrm{m})$. The mobile phase consisted of $20 \mathrm{mM}$ ammonium formate and acetonitrile at a ratio of 60:40. Aliquots of $20 \mu \mathrm{L}$ were injected at a mobile phase flow rate of $0.3 \mathrm{~mL} / \mathrm{min}$. Multiple reaction monitoring was performed in positive or negative modes according to different compounds. The transitions were listed in Table 1. The mass spectra of the metabolites formed in the incubations were identical to those of the corresponding authentic standards, including 7-hydrxoyl coumarin, 1- hydroxyl midazolam, 4- hydroxyl tolbutamide, 4- hydroxyl mephenytoin, $\alpha$ hydroxyl metoprolol, 6- hydroxyl chlorzoxazone [24].

\section{Incubation conditions}

The inhibitory effects of STS on the activities of the CYP isoforms were examined by HLMs (and the expressed CYPs, when required). The CYP isoform-specific probe reactions used were phenacetin O-deethylation (for CYP1A2), coumarin 7-hydroxylation (for CYP2A6), 
Table 1 Transitions and collision energies used in LC-MS/MS for the detection of the probe substrates, metabolites and the internal standard

\begin{tabular}{|c|c|c|c|c|}
\hline Compound name & Ion mode & Precursor ion (m/z) & Product ion (m/z) & Collision energy $(\mathrm{eV})$ \\
\hline Sodium tanshinone IIA sulfonate & Negative & 373 & 358 & 20 \\
\hline Acetaminophen & Positive & 152 & 110 & 15 \\
\hline 7-hydroxyl coumarin & Positive & 162.9 & 107.0 & 20 \\
\hline 1-hydroxyl midazolam & Positive & 342 & 324 & 20 \\
\hline 4-hydroxyl tolbutamide & Positive & 287.0 & 171.0 & 15 \\
\hline 4-hydroxyl mephenytoin & Positive & 235.0 & 150.0 & 10 \\
\hline a-hydroxyl metoprolol & Positive & 284.3 & 116.0 & 20 \\
\hline 6-hydroxyl chlorzoxazone & Positive & 195 & 138 & 20 \\
\hline Irbesartan (IS) & Positive & 429.0 & 206.9 & 22 \\
\hline
\end{tabular}

tolbutamide 4-hydroxylation (for CYP2C9), metoprolol $\alpha$-hydroxylation (for CYP2D6), chlorzoxazone 6-hydroxylation (for CYP2E1), S-mephenytoin 4-hydroxylation (for CYP2C19), and midazolam 1-hydroxylation (for CYP3A4). The incubation mixtures consisted of substrate probe, HLMs $(0.5 \mathrm{mg} / \mathrm{mL})$ or CYP isoforms (10 pmol), and $0.1 \mathrm{M}$ sodium phosphate buffer $(\mathrm{pH} 7.4)$ in a total volume of $0.2 \mathrm{~mL}$ that was pre-warmed for $5 \mathrm{~min}$ at $37^{\circ} \mathrm{C}$ without (control) and with multiple concentrations of STS. Each reaction was initiated by addition of $1 \mathrm{mg} /$ $\mathrm{mL}$ NADPH. The final incubations were performed in a shaking water bath at $37{ }^{\circ} \mathrm{C}$ for $30 \mathrm{~min}$. The incubations were performed in triple, and the incubation conditions specific to each CYP isoform were within the linear range for the velocity of the reaction (incubation time as well as substrate and protein concentrations). STS, probe substrates, and inhibitors were dissolved in methanol, and the final solvent concentration in all incubations (including controls) was $1 \%$. The reactions were stopped by adding $0.2 \mathrm{~mL}$ of ice-cold acetonitrile containing irbesartan $(114.9 \mathrm{ng} / \mathrm{mL})$ as the internal standard. The samples were vortexed for $5 \mathrm{~min}$. After centrifugation (5415D centrifuge, Eppendorf, Germany) (12,000 $\times g$ for $10 \mathrm{~min})$, the supernatants were transferred and aliquots of $20 \mu \mathrm{L}$ were injected into the HPLC-MS/MS system for analysis.

\section{Kinetic analysis of STS}

Kinetic analyses were performed for STS, and the data generated were used as a guide for selecting the appropriate concentrations of STS in the subsequent inhibition experiments. Thus, the kinetic parameters for the metabolism of STS were determined by incubating increasing concentrations of STS $(1-100 \mu \mathrm{M})$ (without inhibitor) at $37{ }^{\circ} \mathrm{C}$ with HLMs $(0.5 \mathrm{mg} / \mathrm{mL})$ or CYP isoforms $(10 \mathrm{pmol})$, and $0.1 \mathrm{M}$ sodium phosphate buffer $(\mathrm{pH}$ 7.4 ) in a total volume of $0.2 \mathrm{~mL}$. The incubation conditions consisted of substrate probe, HLMs $(0.5 \mathrm{mg} / \mathrm{mL})$ or
CYP isoforms (10 pmol), and 0.1 M sodium phosphate buffer ( $\mathrm{pH}$ 7.4) in a total volume of $0.2 \mathrm{~mL}$. The equation for STS reaction velocity $(V)$ by the HLMs or CYP isoforms was expressed as $V=\left(C_{0}-C_{t}\right) / \mathrm{T} / C_{p}$, where $C_{0}$ and $C_{t}$ represent the initial and final concentrations of STS in the incubation solution, respectively, $\mathrm{T}$ is the incubation time ( $\mathrm{min}$ ), and $C_{p}$ is the protein concentration $(\mathrm{mg} / \mathrm{mL}$ or nmol). All values were expressed as the mean \pm standard deviation (SD). The mean intrinsic clearance rate $\left(C L_{\text {int }}\right)$ for the in vitro incubation was estimated by $V_{\text {max }}$ $K_{m}$

\section{Specific CYP isoforms screened for metabolism of STS}

We determined the inhibitory effects of specific inhibitors on the metabolism of STS by HLMs to screen for the specific CYP isoform responsible for STS metabolism. Inhibitors including FUR (CYP1A2 inhibitor; $1 \mu \mathrm{M}$ ), TRA (CYP2A6 inhibitor; $1 \mu \mathrm{M}$ ), SUL (CYP2C9 inhibitor; $1 \mu \mathrm{M})$, QUI (CYP2D6 inhibitor; $1 \mu \mathrm{M})$, CHL (CYP2E1 inhibitor; $5 \mu \mathrm{M}$ ), TIC (CYP2C19 inhibitor; $1 \mu \mathrm{M})$, and KET (CYP3A4 inhibitor; $1 \mu \mathrm{M}$ ) were separately incubated with STS $(10 \mu \mathrm{M})$, HLMs, and NADPH under the same incubation conditions as mentioned above. The concentrations of the inhibitors used were approximately the same as their respective $I C_{50}$ values from a previous report [25]. The inhibitory effects of the specific inhibitors on the metabolic clearance rate (MCR) of STS were evaluated separately to screen for the CYP isoforms responsible for STS metabolism. The relative activity of each CYP isoform was calculated by dividing the peak area of STS incubated with the inhibitor by the peak area of STS in the negative control.

\section{Inhibition studies for $I C_{50}$ determination}

A pilot inhibitory analysis of each CYP isoform was performed to determine the potency of inhibition and to select CYP isoforms for further detailed study of their 
inhibitory activities. Various concentrations of STS $(1-100 \mu \mathrm{M})$ and a single CYP isoform-specific substrate concentration (the $K_{m}$ value) were used to determine the inhibitory effects of STS on specific CYP isoforms. The substrates comprising phenacetin, coumarin, tolbutamide, metoprolol, chlorzoxazone, S-mephenytoin, and midazolam were employed at concentrations of 10, 5, $100,7.5,40,100$, and $5 \mu \mathrm{M}$, respectively [25]. All incubation conditions were the same as those described above. The inhibitory effects on the CYP isoforms were investigated individually by incubating HLMs in the absence or presence of STS. An incubation solution containing only the solvent used to dissolve STS was regarded as the negative control, while solutions containing only the specific inhibitors were regarded as positive controls. The $I C_{50}$ values for STS were determined and compared with those of the specific inhibitors mentioned above (Table 2).

\section{Determination of $K_{i}$}

In pilot experiments $\left(I C_{50}\right.$ determination), STS inhibited CYP3A4, while its effects on the remaining CYPs (CYP1A2, CYP2A6, CYP2C9, CYP2D6, CYP2E1, and CYP2C19) were minimal. Therefore, Dixon plots for the inhibition of CYP3A4 were determined by incubating the substrate probe at multiple concentrations with or without the test inhibitor at multiple concentrations with the HLMs and cofactors. The inhibition data obtained from the pilot experiments were used as a guide to generate appropriate probe substrate and test inhibitor concentrations for determination of the $K_{i}$ values for each CYP isoform. The isoform-specific probe substrate concentrations used were 5-50 $\mu \mathrm{M}$ midazolam for CYP3A4. The STS concentrations used were $0-100 \mu \mathrm{M}$.

\section{Calculation of enzyme kinetics and statistical analysis}

The MCR of the incubation solution without any specific inhibitor for STS was considered to be $100 \%$ to determine the major enzymes responsible for STS metabolism in HLMs. The effects of the specific inhibitors on the MCR of STS were evaluated by one-way analysis of variance (ANOVA) (SPSS Inc., USA), LSD test was used for the homogeneity of variance, otherwise Tamhane's test. Values of $P<0.05$ denoted significance in all cases. The apparent kinetic parameters of STS $\left(K_{m}\right.$ and $\left.V_{\max }\right)$ were determined by fitting the Michaelis-Menten equation using GraphPad Prism Enzyme Kinetic 5 Demo software (GraphPad Co. Ltd., USA). The equation was expressed as $V=V_{\max }[S] /\left(K_{m}+[S]\right)$, where $K_{m}$ is the substrate concentration at which the reaction velocity is $50 \%$ of $V_{\max }$. The activity of each CYP isoform was calculated by the MCR of its corresponding probe substrate to determine the inhibition of the CYP isoforms. The MCR of the probe substrate was considered to be $100 \%$ when no specific inhibitor and STS were added to the incubation assay. The $I C_{50}$ values were determined by analyzing the plot of the logarithm of the inhibitor concentration versus the percentage of activity remaining after inhibition, using the SPSS for Windows Version 11.5 (SPSS Inc., USA). The inhibition data were fitted to different models of enzyme inhibition (competitive, noncompetitive, and uncompetitive) by nonlinear least-squares regression analysis using GraphPad Prism 5 software (GraphPad Co. Ltd., USA) to calculate the $K_{i}$ values.

\section{Results}

\section{Kinetic analysis of STS}

The metabolism of STS after incubation with HLMs and CYP3A4 was shown in Fig. 2a, b. The $K_{m}$ and $V_{\max }$

Table $2 I C_{50}$ and $K_{i}$ values of STS against human CYP isoforms compared with that of specific inhibitors reported in literature

\begin{tabular}{|c|c|c|c|c|c|}
\hline \multirow[t]{2}{*}{ CYP } & \multirow[t]{2}{*}{ Activity } & \multicolumn{2}{|l|}{$I C_{50}(\mu \mathrm{M})$} & \multicolumn{2}{|l|}{$K_{i}(\mu \mathrm{M})$} \\
\hline & & STS & $\begin{array}{l}\text { Specific inhibitor/ } \\
\text { reported values }^{\mathrm{a}}\end{array}$ & STS & $\begin{array}{l}\text { Specific inhibitor/ } \\
\text { reported values }^{\mathrm{a}}\end{array}$ \\
\hline CYP1A2 & Phenacetin O-deethylation & $>100$ & FUR/1.4 [23] & - & FUR/3 [21] \\
\hline CYP2A6 & Coumarin 7-hydroxylation & $>100$ & TRA/0.42 $\pm 0.07[24]$ & - & TRA/0.17 [24] \\
\hline CYP2C9 & Tolbutamide 4-hydroxylation & $>100$ & SUL/0.3-1.5 [23, 25] & - & SUL/0.3 [21] \\
\hline CYP2D6 & Metoprolol a-hydroxylation & $>100$ & QUI/0.02-0.68 [23, 25] & - & SUL/0.027-0.4 [21, 26, 27] \\
\hline CYP2E1 & Chlorzoxazone 6-hydroxylation & $>100$ & $\mathrm{DIE} / 21.30[23]$ & - & $\mathrm{CHL} / 12$ [28] \\
\hline CYP2C19 & S-Mephenytoin 4-hydroxylation & $>100$ & $\mathrm{TCL} / 0.52-1.6[25]$ & - & $\mathrm{TCL} / 1.2 \pm 0.5[21]$ \\
\hline CYP3A4 & Midazolam 1-hydroxylation & $6.377(5.536,7.347)^{b}$ & KET/0.08-0.24 [21] & $3.183(0.184,6.95)^{b}$ & KET/0.015 [21] \\
\hline
\end{tabular}

"-" Represents the data that is not determined

STS sodium tanshinone II A sulfonate; FUR furafylline; TRA trans-2-phenylcyclopropylamine hydrochloride; SUL sulfaphenazole; QUI quinidine; CHL chlormethiazole hydrochloride; TIC ticlopidine hydrochloride; KET ketoconazole; DIE diethyldithiocarbamate

a $I_{50}$ and $K_{i}$ values of specific inhibitors were referred to the reported literatures

b Represents $95 \%$ confidence interval 


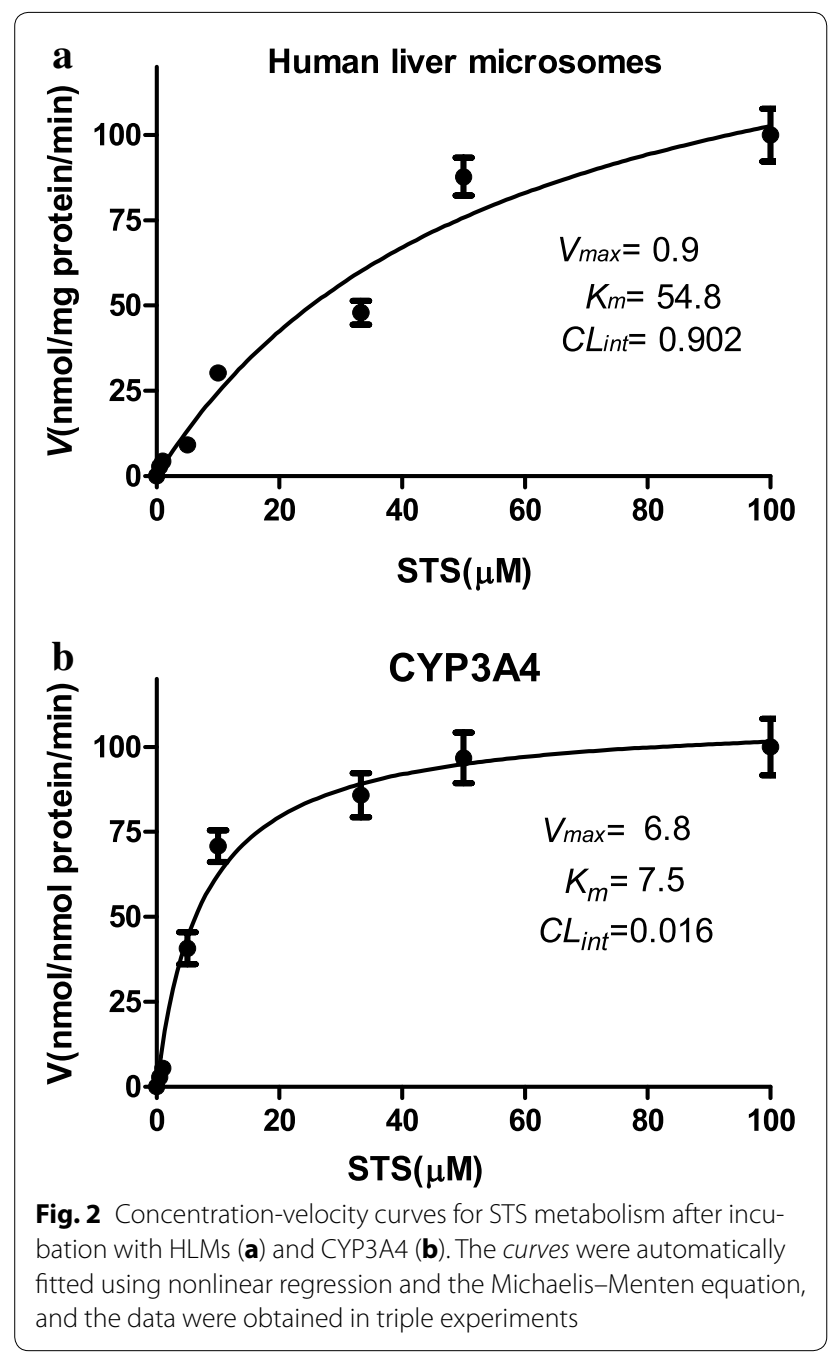

values for HLMs and CYP3A4 were $54.8 \pm 14.6 \mu \mathrm{M}$ and $0.9 \pm 0.1 \mathrm{nmol} / \mathrm{mg}$ protein $/ \mathrm{min}$, respectively, and $7.5 \pm 1.4 \mu \mathrm{M}$ and $6.8 \pm 0.3 \mathrm{nmol} / \mathrm{nmol} \mathrm{P} 450 / \mathrm{min}$, respectively. The in vitro $C L_{\text {int }}$ values for STS with HLMs and CYP3A4 were $0.016 \mathrm{~mL} / \mathrm{mg}$ protein/min and $0.902 \mathrm{~mL} /$ nmol P450/min, respectively.

\section{Specific CYP isoforms for the metabolism of STS}

The inhibitory effects of the CYP specific inhibitors on the MCR of STS in HLMs were shown in Fig. 3. The concentrations of FUR, TRA, SUL, QUI, TIC, and KET were $1 \mu \mathrm{M}$, while that of CHL was $5 \mu \mathrm{M}$. The concentrations were selected on the basis of previously reported $I C_{50}$ or $K_{i}$ values for the CYP isoforms to ensure adequate inhibitory selectivity, and maximal inhibitory potency [26-29]. In the presence of KET $(1 \mu \mathrm{M})$, the MCR of STS decreased to $37.4 \%$ of that of the control. However, the other inhibitors had no obvious inhibitory effects

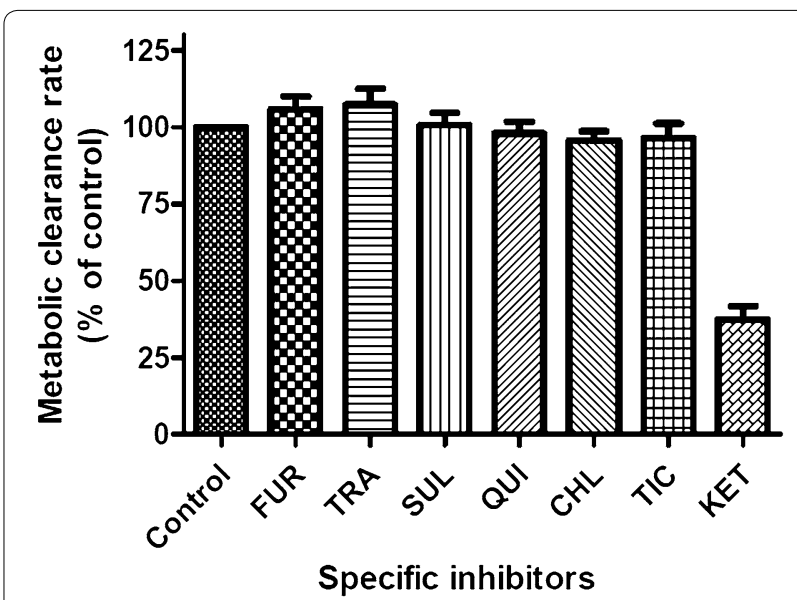

Fig. 3 Effects of specific inhibitors on CYP-mediated STS (10 $\mu \mathrm{M})$ metabolism in HLMs. The concentration of CHL was $5 \mu \mathrm{M}$, while that for the other specific inhibitors was $1 \mu \mathrm{M}$. Each data point represents the mean \pm SD of triple determinations $(n=3)$. In the presence of KET $(1 \mu M)$, the MCR of STS decreased to $27.28 \%$ of the control value, while the other inhibitors had no significant inhibitory effects on the metabolism of STS. FUR furafylline, specific inhibitor of CYP1A2; TRA trans-2-phenylcyclopropylamine, specific inhibitor of CYP2A6; SUL sulfaphenazole, specific inhibitor of CYP2C9; QUI quinidine, specific inhibitor of CYP2D6; CHL: chlormethiazole, specific inhibitor of CYP2E1; T/Cticlopidine, specific inhibitor of CYP2C19; KET ketoconazole, specific inhibitor of CYP3A4

on the metabolism of STS. The effects of the screened enzymes were further confirmed with human recombinant CYPs using specific inhibitors, and the MCR of STS was decreased to $58.4 \%$ (STS, $10 \mu \mathrm{M}$ ) and $29.4 \%$ (STS, $50 \mu \mathrm{M}$ ) of the control value for CYP3A4 (Fig. 4), indicating that CYP3A4 was the major enzyme responsible for the metabolism of STS in vitro.

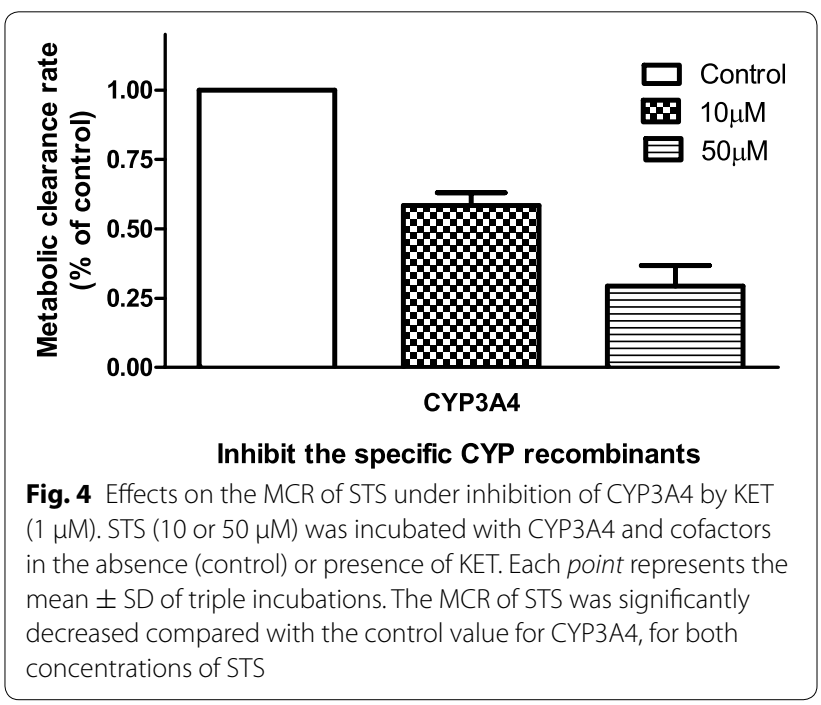




\section{Estimation of $I C_{50}$ and $K_{i}$ values}

The inhibitory effects of multiple concentrations of STS $(1-100 \mu \mathrm{M})$ on the activity of each CYP isoform were determined by metabolism of a single concentration of isoform-specific probe with HLMs (or expressed CYPs, when needed). STS showed potent inhibition of CYP3A4 (midazolam 1-hydroxylation), with an $I C_{50}$ of $6.4 \mu \mathrm{M}$. The inhibitory effects of STS on the activities of CYP1A2, CYP2A6, CYP2C9, CYP2D6, CYP2E1, and CYP2C19 were negligible (Fig. 5); therefore, we did not calculate the $I C_{50}$ or $K_{i}$ values for these enzymes related to STS.

\section{Discussion}

According to Jeong et al. [30], $I C_{50}$ values are qualitatively informative and helpful for addressing whether inhibition has occurred, but are of limited quantitative use because they can be influenced by the substrate concentration selected. Hence, it might not be accurate to use these parameters for quantitative prediction of drug interactions in vivo. Therefore, we performed additional experiments designed to estimate the $K_{i}$ values. The preliminary inhibition data generated from a single probe substrate reaction were used to simulate the appropriate range of substrate and inhibitor concentrations for use in the construction of Dixon plots for inhibition of the CYP isoforms by STS in HLMs, from which precise $K_{i}$ values were estimated.

For CYP3A4, the $K_{i}$ values were determined by midazolam as the probe substrate. Among all of the CYPs
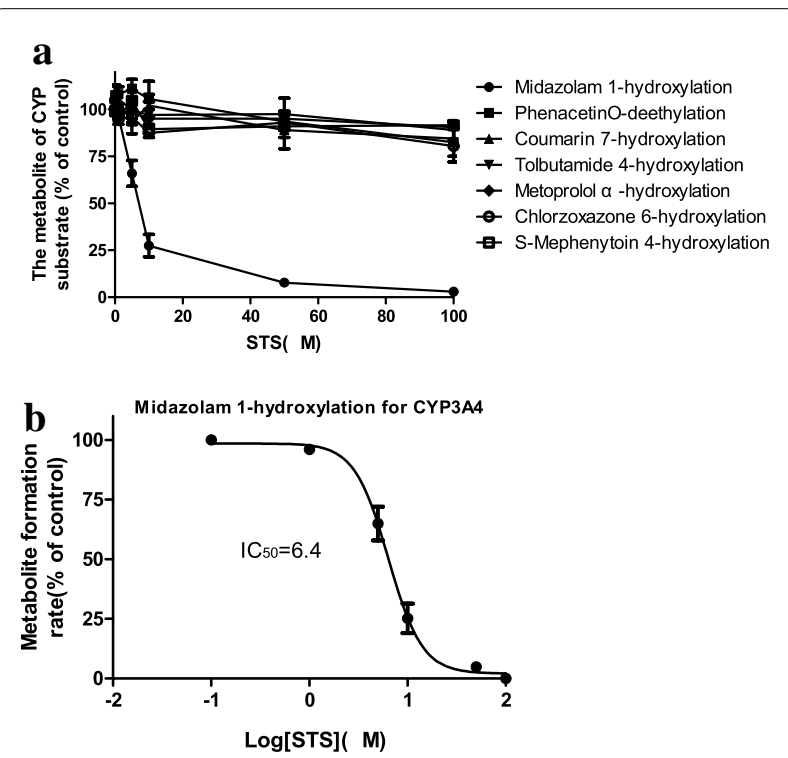

Fig. 5 Effects of STS on the metabolic reactions of the seven CYP specific substrates in $\mathrm{HLMs}(\mathbf{a})$ and the representative $/ C_{50}$ plots of STS on midazolam 1-hydroxylation for CYP3A4 (b). Each data point represents the mean \pm SD of triple determinations tested, CYP3A4 was the most sensitive to inhibition by STS (Table 2). Representative Dixon plots for the inhibition of CYP3A4 in HLMs were shown in Fig. 6. Visual inspection of the Dixon plots and further analysis of the parameters of the enzyme inhibition models suggested that the inhibition data fitted well to a competitive type of inhibition (Fig. 7). The $K_{i}$ values estimated by a nonlinear regression model for competitive enzyme inhibition of CYP3A4-catalyzed midazolam 1-hydroxylation in HLMs were less than $5 \mu \mathrm{M}$ (Table 2, Fig. 7).

According to Kong et al. [31], the potency of a test compound could be classified according to its $I C_{50}$ values, as follows: potent, if $I C_{50}$ is $\leq 20 \mu \mathrm{g} / \mathrm{mL}$ or $\leq 10 \mu \mathrm{M}$; moderate, if $I C_{50}$ is $20-100 \mu \mathrm{g} / \mathrm{mL}$ or $10-50 \mu \mathrm{M}$; and weak, if $I C_{50}$ is $\geq 100 \mu \mathrm{g} / \mathrm{mL}$ or $\geq 50 \mu \mathrm{M}$. Thus, STS was a potent inhibitor for CYP3A4 and a weak inhibitor for the other six CYPs tested in this study.

Although STS was a potent inhibitor of CYP3A4, the $I C_{50}$ value of CYP3A4 for midazolam 1-hydroxylation was 26.6-79.7-fold higher and the $K_{i}$ value was approximately 212-fold higher than that of KET in HLMs compared with a previous study [26] (Table 2), indicating that the inhibitory effect of STS on CYP3A4 was much less than that of KET.
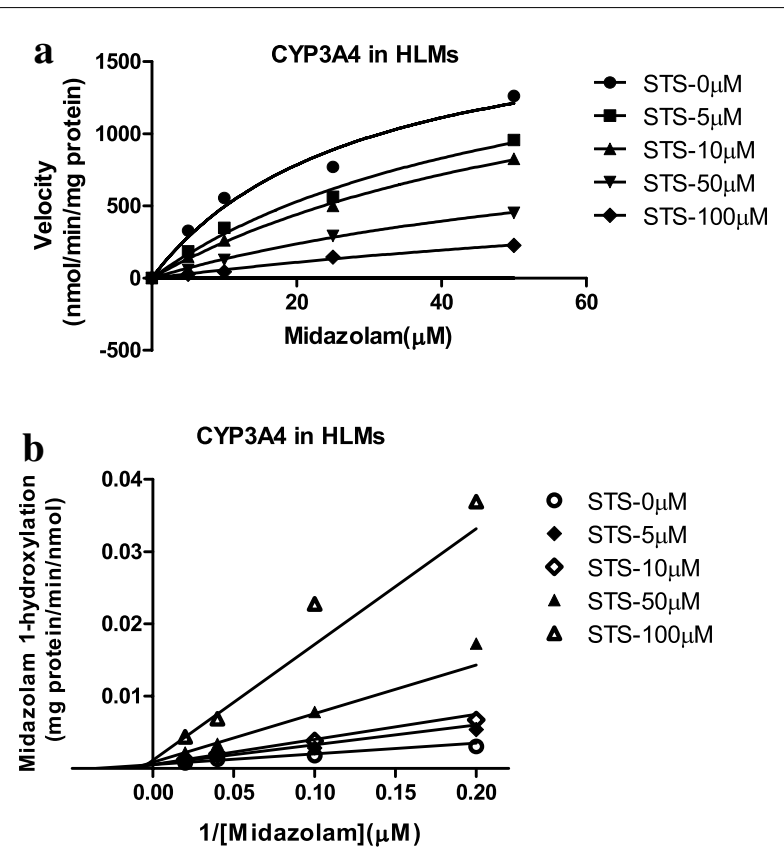

Fig. 6 Non-linear regression and double reciprocal (LineweaverBurk) plots for direct inhibition of midazolam 1-hydroxylation by different concentrations of STS $(0-100 \mu \mathrm{M})$ in HLM incubations ( $0.5 \mathrm{mg}$ protein $/ \mathrm{mL}$ ). The inhibition of CYP3A4 activity by STS can be best-described as full competitive inhibition. Each data point is the mean of triple incubations 


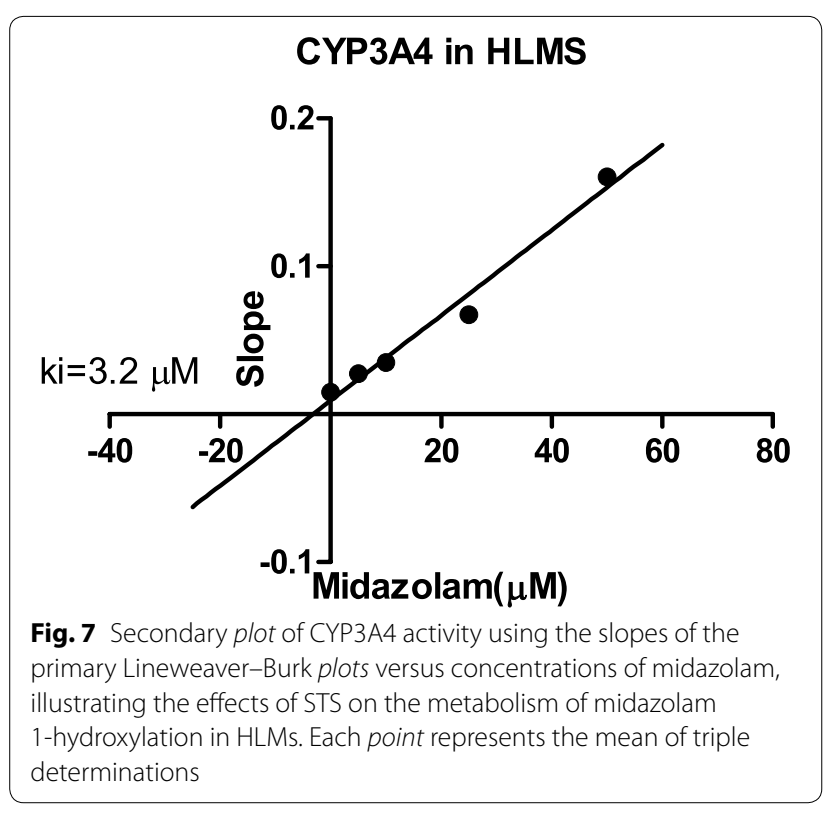

Although the molecular structural difference between STS and tanshinone IIA is only the presence of a sulfonic acid group bond at the $\mathrm{C}-16$ position [22], it resulted in a difference in inhibition of CYP activity. Wang et al. [32] reported that tanshinone IIA was a potent competitive CYP1A2 inhibitor with $K_{i}$ values of $1.45 \mu \mathrm{M}$ for pooled HLMs and $0.05 \mu \mathrm{M}$ for a specific human CYP1A2 isoform, and a medium competitive inhibitor of CYP2C9. In this study, STS was a potent competitive CYP3A4 inhibitor with a $K_{i}$ value of $3.2 \mu \mathrm{M}$ for pooled HLMs, but was not an inhibitor of CYP1A2.

The CYP3A4 enzyme, one of the dominant CYP enzymes in both the liver and extrahepatic tissues such as the intestine, plays an important role in the oxidation of xenobiotics and contributes to the biotransformation of about $60 \%$ of currently used therapeutic drugs [33]. Human CYP3A4 is one of the most abundant drugmetabolizing P450 isoforms in HLMs, and accounts for approximately $40 \%$ of the total P450 activity [32]. Because STS was a potent inhibitor of CYP3A4 and given that CYP3A4 is responsible for the metabolism and disposition of a large number of currently used drugs, the potential herb-drug interactions of STS with drugs that were substrates of CYP3A4 or drugs with a narrow therapeutic index could not be negligible in the clinic. The present study supports the notion that STS is a substrate of CYP3A4 alone, while the issue of whether STS may be metabolized by other enzyme systems, such as more complex in vitro systems (hepatocytes for instance) that are not present (or functional in microsomes) remains unclear. Therefore, the present data do not support that
STS would be a victim of drug-drug interactions (DDIs) by other CYP3A4 inhibitors, and further studies are required to elucidate this point.

Although STS in the form of injections is often used in clinical settings, pharmacokinetics studies in humans were lacking. Meanwhile, (Additional file 1) pharmacokinetics studies $[15,16]$ of STS in rats indicated that STS was widely distributed in most tissues after intravenous administration $(2 \mathrm{mg} / \mathrm{kg})$, and that it was mainly cleared via both the liver and kidney. The maximal STS concentration $(>10 \mu \mathrm{g} / \mathrm{mL})$ was found in the liver at $5 \mathrm{~min}$ after drug administration, subsequently declined progressively during $30 \mathrm{~min}$, and then decreased quickly over time thereafter. STS could be determined at 12 and $4 \mathrm{~h}$ after drug administration in the liver and kidney, respectively. STS was hardly detected at $2 \mathrm{~h}$ after drug administration in other tissues $[15,16]$. Therefore, co-administered medicines were suggested to be given at least $2 \mathrm{~h}$ after STS administration to avoid the risk of DDIs with STS.

Genetic variations in the CYP3A4 gene may influence the level or function of the CYP3A4 protein, and more than 30 single nucleotide polymorphisms (SNPs) have been identified in the CYP3A4 gene [34]. In previous studies, allelic variants in the gene encoding CYP3A4 were shown to affect enzyme activities, such as observations that variant CYP3A4 forms T363 M (<40\%) and T185S $(<60 \%)$ reduced testosterone $6 \beta$ - and $2 \beta$-hydroxylase activities compared with the wild-type enzyme [35], while some SNPs in the CYP3A4 gene such as CYP3A4*1B and CYP3A4*22 were reported to affect the pharmacokinetics of tacrolimus [36]. However, the issue of whether genetic polymorphisms of CYP3A4 can affect the DDIs of STS requires further study for clarification in the future.

\section{Conclusion}

This in vitro study showed that STS mainly inhibited the activities of CYP3A4.

\section{Additional file}

Additional file 1. The post-hoc test for the effects of the specific inhibitors on the MCR of STS.

\section{Abbreviations}

STS: sodium tanshinone IIA sulfonate; CYPS: cytochrome P450 enzymes; HLMs: human liver microsomes; NADPH: $\beta$-nicotinamide adenine dinucleotide 2'-phosphate reduced tetrasodium salt hydrate; FUR: furafylline; TRA: trans2-phenylcyclopropylamine hydrochloride; KET: ketoconazole; SUL: sulfaphenazole; QUI: quinidine; CHL: chlormethiazole hydrochloride; TIC: ticlopidine hydrochloride; MCR: metabolic clearance rate.

\section{Authors' contributions}

YC and DSOY designed the study and performed the data analysis. YC, WHH, DC, WZ, ZRT and JX conducted the acquisition of data. YC, JBP, YCW, YG, 
DLH, JX and DSOY conducted the analysis and interpretation of data. YC and DSOY wrote the manuscript and revised the manuscript. All authors read and approved the final manuscript.

\section{Author details \\ 1 Department of Clinical Pharmacology, Xiangya Hospital, Central South University, 110 Xiangya Road, Changsha 410078, Hunan, China. ${ }^{2}$ Institute of Clinical Pharmacology, Central South University, 110 Xiangya Road, Chang- sha 410078 , Hunan, China. ${ }^{3}$ Department of Pharmacy, Xiangya Hospital, Central South University, 87 Xiangya Road, Changsha 410008, Hunan, China.}

\section{Acknowledgements}

This work was supported by the National Scientific Foundation of China (No. 81302850, 81300204), Financial Grant from the China Postdoctoral Science Foundation (No. 2013M531817, 2014T70793 and 2013M542146), Science and Technology Plan Projects of Hunan Province (2014RS4011), Key Laboratory Funds of Hunan Province (13K003), Changsha Science and technology key project (K1406004-31, K1308032-31), and the Fundamental Research Funds for the Central South University (No. 1681-7608040003). The funders had no role in the study design, data collection and analysis, decision to publish, or preparation of the manuscript.

\section{Competing interests}

The authors declare that they have no competing interests.

Received: 21 August 2014 Accepted: 14 March 2016

Published online: 22 March 2016

\section{References}

1. Gao S, Liu Z, Li H, Little PJ, Liu P, Xu S. Cardiovascular actions and therapeutic potential of tanshinone IIA. Atherosclerosis. 2012;220:3-10.

2. Li W, Zhang Y, Xing C, Zhang M. Tanshinone IIA represses inflammatory response and reduces radiculopathic pain by inhibiting IRAK-1 and NF-KB/p38/JNK signaling. Int Immunopharmacol. 2015;28:382-9.

3. Liu F, Yu G, Wang G, Liu H, Wu X, Wang Q, Liu M, Liao K, Wu M, Cheng X, Hao H. An NQO1-initiated and p53-independent apoptotic pathway determines the anti-tumor effect of tanshinone IIA against non-small cell lung cancer. PLoS One. 2012;7:e42138.

4. Hu H, Zhai C, Qian G, Gu A, Liu J, Ying F, Xu W, Jin D, Wang H, Hu H, Zhang $Y$, Tang $G$. Protective effects of tanshinone IIA on myocardial ischemia reperfusion injury by reducing oxidative stress, HMGB1 expression, and inflammatory reaction. Pharm Biol. 2015;53:1752-8.

5. Wang P, Zhou S, Xu L, Lu Y, Yuan X, Zhang H, Li R, Fang J, Liu P. Hydrogen peroxide-mediated oxidative stress and collagen synthesis in cardiac fibroblasts: blockade by tanshinone IIA. J Ethnopharmacol. 2013;145:152-61.

6. Maione F, De Feo V, Caiazzo E, De Martino L, Cicala C, Mascolo N. Tanshinone IIA, a major component of Salvia milthorriza Bunge, inhibits platelet activation via Erk-2 signaling pathway. J Ethnopharmacol. 2014;155:1236-42.

7. Maione F, Cantone V, Chini MG, De Feo V, Mascolo N, Bifulco G. Molecular mechanism of tanshinone IIA and cryptotanshinone in platelet antiaggregating effects: an integrated study of pharmacology and computational analysis. Fitoterapia. 2015;100:174-8.

8. Yu H, Subedi RK, Nepal PR, Kim YG, Choi HK. Enhancement of solubility and dissolution rate of cryptotanshinone, tanshinone I and tanshinone IIA extracted from Salvia miltiorrhiza. Arch Pharm Res. 2012;35:1457-64.

9. Long R, You Y, Li W, Jin N, Huang S, Li T, Liu K, Wang Z. Sodium tanshinone IIA sulfonate ameliorates experimental coronary no-reflow phenomenon through down-regulation of FGL2. Life Sci. 2015;142:8-18.

10. Mao S, Wang L, Zhao X, Shang H, Zhang M, Hinek A. Sodium tanshinone IIA sulfonate for reduction of periprocedural myocardial injury during percutaneous coronary intervention (STAMP trial): rationale and design. Int J Cardiol. 2015;182:329-33.

11. Yang L, Hu J, Hao HZ, Yin Z, Liu G, Zou XJ. Sodium tanshinone IIA sulfonate attenuates the transforming growth factor- $\beta 1$-induced differentiation of atrial fibroblasts into myofibroblasts in vitro. Int J Mol Med. 2015;35:1026-32.
12. Zhang JQ, Gu YL, Wu CH, Ma HL, He JP, Bai YS, Li HQ. Effects of sodium tanshinone IIA sulfonate against Marek's disease virus in experimentally infected chickens. Int J Biol Macromol. 2013;58:258-62.

13. Wang J, Sun P, Feng Y, Guo J, Sun Y, Lei H, Xu J, Li H. Sodium tanshinone IIA sulfonate affects Marek's disease virus replication by inhibiting $g B$ expression. Pharm Biol. 2016;54:701-4.

14. Sun N, Cong XM, Jiang JB, Zhao JX, Wang WK, Duan ZB, Hu YL, Lei HM, $\mathrm{Li} \mathrm{HQ}$. Sodium tanshinone IIA sulfonate inhibits the meq, $u 149$ and VP22 expression of Marek's disease virus. Antivir Ther. 2014;19:793-8.

15. Mao S, Jin H, Bi Y, Liang Z, Li H, Hou S. Ion-pair reversed-phase HPLC method for determination of sodium tanshinone IIA sulfonate in biological samplesand its pharmacokinetics and biodistribution in mice. Chem Pharm Bull. 2007:55:753-6.

16. Hao H, Wang G, Cui N, Li J, Ding Z. Determination of sodium tanshinone IIA sulfonate in plasma by liquid chromatography-electrospray ionisationtandem mass spectrometry. Biomed Chromatogr. 2007;21:1172-9.

17. Zhou S, Gao Y, Jiang W, Huang M, Xu A, Paxton JW. Interactions of herbs with cytochrome P450. Drug Metab Rev. 2003;35:35-98.

18. Yang XF, Wang NP, Lu WH, Zeng FD. Effects of Ginkgo biloba extract and tanshinone on cytochrome P-450 isozymes and glutathione transferase in rats. Acta Pharmacol Sin. 2003;24:1033-8.

19. Ueng YF, Kuo YH, Peng HC, Chen TL, Jan WC, Peter Guengerich F, Lin YL. Diterpene quinone tanshinone IIA selectively inhibits mouse and human cytochrome p4501A2. Xenobiotica. 2003;33:603-13.

20. Ueng YF, Kuo YH, Wang SY, Lin YL, Chen CF. Induction of CYP1A by a diterpene quinone tanshinone IIA isolated from a medicinal herb Salvia miltiorrhiza in C57BL/6 J but not in DBA/2 J mice. Life Sci. 2004;74:885-96.

21. Liu HX, Hu Y, Liu Y, He YQ, Li W, Yang L. Hydroxylation of tanshinone Ila in human liver microsomes is specifically catalysed by cytochrome P4502A6. Xenobiotica. 2009;39:382-90.

22. Chen S, Zhao X, Li Y, Yang D, Zhou T, Fan G. Impurities preparation of sodium tanshinone IIA sulfonate by high-speed counter-current chromatography and identification by liquid chromatography/multistage tandem mass spectrometry. J Chromatogr A. 2013;1288:28-34.

23. Chen Y, Tu JH, He YJ, Zhang W, Wang G, Tan ZR, Zhou G, Fan L, Zhou HH. Effect of sodium tanshinone II A sulfonate on the activity of CYP1A2 in healthy volunteers. Xenobiotica. 2009;39:508-13.

24. Huang X, Guo Y, Huang WH, Zhang W, Tan ZR, Peng JB, Wang YC, Hu DL, Ouyang DS, Xiao J, Wang Y, Luo M, Chen Y. Searching the cytochrome p450 enzymes for the metabolism of meranzin hydrate: a prospective antidepressant originating from Chaihu-Shugan-San. PLoS One. 2014;9:e113819.

25. Xu C, Desta Z. In vitro analysis and quantitative prediction of efavirenz inhibition of eight cytochrome P450 (CYP) enzymes: major effects on CYPs 2B6, 2C8, 2C9 and 2C19. Drug Metab Pharmacokinet. 2013;28:362-71.

26. Bourrie M, Meunier V, Berger Y, Fabre G. Cytochrome P450 isoform inhibitors as a tool for the investigation of metabolic reactions catalyzed by human liver microsomes. J Pharmacol Exp Ther. 1996;277:321-32.

27. Li X, Wang K, Wei W, Liu YY, Gong L. In vitro metabolism of brucine by human liver microsomes and its interactions with CYP substrates. Chem Biol Interact. 2013;204:140-3.

28. Taavitsainen $\mathrm{P}$, Juvonen $\mathrm{R}$, Pelkonen $\mathrm{O}$. In vitro inhibition of cytochrome P450 enzymes in human liver microsomes by a potent CYP2A6 inhibitor trans-2-phenylcyclopropylamine (tranylcypromine), and its nonamine analog, cyclopropylbenzene. Drug Metab Dispos. 2001;29:217-22.

29. Qin CZ, Ren X, Tan ZR, Chen Y, Yin JY, Yu J, Qu J, Zhou HH, Liu ZQ. A high-throughput inhibition screening of major human cytochrome P450 enzymes using an in vitro cocktail and liquid chromatography-tandem mass spectrometry. Biomed Chromatogr. 2014;28:197-203.

30. Jeong S, Nguyen PD, Desta Z. Comprehensive in vitro analysis of voriconazole inhibition of eight cytochrome P450 (CYP) enzymes: major effect on CYPs 2B6, 2C9, 2C19, and 3A. Antimicrob Agents Chemother. 2009;53:541-51.

31. Kong WM, Chik Z, Ramachandra M, Subramaniam U, Aziddin RE, Mohamed Z. Evaluation of the effects of Mitragyna speciosa alkaloid extract on cytochrome P450 enzymes using a high throughput assay. Molecules. 2011;16:7344-56

32. Wang $X$, Cheung CM, Lee WY, Or PM, Yeung JH. Major tanshinones of Danshen (Salvia miltiorrhiza) exhibit different modes of inhibition on 
human CYP1A2, CYP2C9, CYP2E1 and CYP3A4 activities in vitro. Phytomedicine. 2010;17:868-75.

33. Haddad A, Davis M, Lagman R. The pharmacological importance of cytochrome CYP3A4 in the palliation of symptoms: review and recommendations for avoiding adverse drug interactions. Support Care Cancer. 2007;15:251-7.

34. Zhou LP, Yao F, Luan H, Wang YL, Dong XH, Zhou WW, Wang QH. CYP3A4*1B polymorphism and cancer risk: a HuGE review and metaanalysis. Tumour Biol. 2013;34:649-60.
35. Murayama N, Nakamura T, Saeki M, Soyama A, Saito Y, Sai K, Ishida S, Nakajima O, Itoda M, Ohno Y, Ozawa S, Sawada J. CYP3A4 gene polymorphisms influence testosterone 6beta-hydroxylation. Drug Metab Pharmacokinet. 2002;17:150-6.

36. Gervasini G, Garcia M, Macias RM, Cubero JJ, Caravaca F, Benitez J. Impact of genetic polymorphisms on tacrolimus pharmacokinetics and the clinical outcome of renal transplantation. Transplant Int. 2012;25:471-80.
Submit your next manuscript to BioMed Central and we will help you at every step:

- We accept pre-submission inquiries

- Our selector tool helps you to find the most relevant journal

- We provide round the clock customer support

- Convenient online submission

- Thorough peer review

- Inclusion in PubMed and all major indexing services

- Maximum visibility for your research

Submit your manuscript at www.biomedcentral.com/submit
() BioMed Central 